\title{
Alocação do tempo entre os gêneros e o mercado de trabalho: uma análise entre casados e solteiros para o Brasil
}

Allocation of time between genders and the labor market: an analysis between Married and single to Brazil

\author{
Daniel de Abreu Pereira Uhr (1) \\ Regis Augusto Ely (2) \\ Renata Pereira Cardoso (3) \\ Júlia Gallego Ziero Uhr (4)
}

\author{
(1) Universidade Federal de Pelotas \\ (2) Universidade Federal de Pelotas \\ (3) Pontifícia Universidade Católica do \\ Rio Grande do Sul \\ (4) Universidade Federal de Pelotas
}

\begin{abstract}
This paper analyzes the differences of wage and time allocation between married and single individuals in Brazil in order to understand how marriage is associated with the wage distribution, the hours of work and the time spent in domestic labor of men and women. For that purpose, we use data from the National Household Sample Survey (PNAD) for the year 2015 and we employ three different estimation methods for complex surveys: ordinary least squares, Propensity Score Weighting, and Propensity Score Matching. The results indicate that married men have higher wage and work hours than single men, whereas married women increase their likelihood of performing domestic labor, as well as the hours spent in such activities. We also find evidence that the time allocation between men and women is affected by the comparative advantage of the couple.
\end{abstract}

\section{Keywords}

marriage, labor, housework, comparative advantage, gender.

JEL Codes J12, J22, D13.

\section{Resumo}

Este artigo analisa as diferenças de salário e alocação de tempo entre casados e solteiros no Brasil, com o objetivo de entender como o casamento está associado à distribuição de salários, jornada e tarefa doméstica dos homens e mulheres. Utilizamos dados da Pesquisa Nacional por Amostra de Domicílios (PNAD) para o ano de 2015 e aplicamos três diferentes métodos de estimação para amostras complexas: minimos quadrados ordinários, Propensity Score Weighting e Propensity Score Matching. Os resultados indicam que homens casados apresentam salário e jornada de trabalho consistentemente superiores aos homens solteiros, enquanto mulheres casadas aumentam sua probabilidade de exercer tarefa doméstica, bem como a jornada de trabalho doméstico. Também encontramos evidências de que a alocação de tempo dos cônjuges em relação ao mercado de trabalho é afetada pela vantagem comparativa do casal.

\section{Palavras-chave}

casamento; trabalho; jornada doméstica; vantagem comparativa; gênero.

Códigos JEL J12, J22, D13. 


\section{Introdução}

A literatura demonstra que o casamento apresenta importantes implicações na alocação do tempo entre os gêneros, principalmente no que diz respeito a divisão entre oferta de trabalho e tarefas domésticas (Kleven et al., 2018; Queiroz; Aragon, 2015; Del Boca; Flinn, 2013). Além disso, alterações nos padrões conjugais trazem diversos impactos à sociedade, como no número de nascimentos, no crescimento da população, na participação das mulheres no mercado de trabalho, na desigualdade de renda, entre outras questões com grande impacto econômico (Becker, 1973).

Este artigo analisa as diferenças entre casados e solteiros no salário e na alocação de tempo, a fim de entender como o casamento pode afetar a distribuição de salários e a jornada de trabalho externa e doméstica entre homens e mulheres. Esta análise compreende variáveis como o salário principal, o salário de todos os trabalhos, a jornada semanal do trabalho principal, a jornada semanal de todos os trabalhos, a probabilidade de ter mais de um emprego, a probabilidade de exercer atividades domésticas e a jornada de trabalho doméstico. Também verificamos se a divisão do trabatho na unidade familiar se altera significativamente quando as esposas auferem salários superiores aos de seus respectivos cônjuges, possivelmente refletindo uma vantagem comparativa no mercado de trabalho obtida pelo cônjuge mais produtivo.

Para esses propósitos utilizamos os dados da Pesquisa Nacional por Amostra de Domicílios (PNAD) para o ano de 2015 e construídas duas amostras distintas. Uma contendo apenas indivíduos casados e outra restringindo os dados para incluir apenas os homens com esposas que exercem atividade no mercado de trabalho. Na primeira, a variável de interesse identifica se o indivíduo possui cônjuge. Na segunda, a variável identifica se a esposa possui remuneração maior do que o marido. Realizamos as estimativas através de Mínimos Quadrados Ordinários e modelos de escore de propensão. Como a PNAD é uma amostra complexa, essa estrutura deve ser considerada para a correta identificação econométrica. Assim, utilizamos as recomendações recentes na literatura sobre a aplicação de métodos de escore de propensão para amostras complexas (Austin et al., 2016; DuGoff et al., 2014). Três modelos foram estimados para cada variável de interesse. O primeiro é uma regressão linear ponderada pelos pesos amostrais complexos (OLS). O segundo é o propensity score weighting (PSW), 
utilizando a multiplicação dos pesos da amostra complexa pelos pesos do escore de propensão. O terceiro é o propensity score matching (PSM), onde utiliza-se o peso da amostra complexa após o pareamento através do escore de propensão.

Esta pesquisa contribui para a literatura de diferenciais salariais e de alocação de tempo entre os gêneros principalmente por analisar empiricamente as diferenças entre casados e solteiros na divisão das tarefas produtivas e domésticas para cada gênero no Brasil. Além disso, ela emprega métodos econométricos recentemente sugeridos pela literatura especializada, de modo a minimizar o viés das estimativas para amostras complexas através do escore de propensão.

A motivação para este estudo está relacionada ao fato de que alterações nos padrões de alocação do tempo por parte dos cônjuges trazem diversos efeitos à sociedade, por exemplo, no número de nascimentos e na educação dos filhos, cujos impactos serão sentidos no futuro. Adicionalmente, existe a preocupação de que a divisão das tarefas seja baseada puramente no gênero e não na produtividade, o que impactaria na participação das mulheres no mercado de trabalho, na desigualdade de renda entre os gêneros, entre outras questões com grande relevância político-econômica.

Quanto à estrutura da pesquisa, além desta introdução, a seção dois apresenta a revisão da literatura. Na seção três descrevemos os dados utilizados, o processo de filtragem, a elaboração das variáveis e a estratégia empírica. Na seção quatro comentamos os resultados. E, por fim, apresentamos as considerações finais.

\section{Revisão da literatura}

Em termos gerais, o casamento é entendido como um contrato de longo prazo onde os cônjuges dividirão a mesma moradia formando um núcleo familiar ${ }^{1}$ (Becker, 1973). E o núcleo familiar tem o propósito de produção e consumo conjunto. Em suma, o modelo econômico do casamento ${ }^{2}$ apresenta quatro fontes de ganho material (Weiss, 1997; Browning et al., 2014). A primeira é a divisão do trabalho a fim de tirar proveito da vantagem com-

1 Para uma breve resenha em português, ver Balbinotto Neto (1992).

2 A teoria que estuda o casamento também é conhecida na literatura econômica como "Family Economics" (Browning et al., 2014). 
parativa ou dos retornos decrescentes. A formação da família proporciona aos seus membros a opção de utilizar melhor o capital humano. Assim, o casal pode avaliar em termos de vantagem comparativa e alocar de forma apropriada quem ofertará trabalho no mercado e quem cuidará das tarefas domésticas ou se os dois farão ambas as tarefas. Existe evidência dessa divisão do trabalho em que homens casados trabalham mais horas no mercado e tem salários substancialmente maiores que homens não casados. Além disso, existe evidência de que mulheres casadas têm salários menores e trabalham mais em casa em tarefas domésticas que mulheres não casadas (Gronau, 1986; Korenman; Neumark, 1991; Man et al., 2011; Sayer, 2010).

A segunda função da formação do núcleo familiar é aumento do crédito e melhor coordenação dos investimentos. $O$ aumento do crédito se dá pela soma das remunerações do trabalho dos indivíduos, e este será aplicado de forma a otimizar a renda da família no tempo. Como a educação é o principal componente da produtividade, a família tende a investir mais na escolaridade da pessoa com maior taxa de retorno, por exemplo, a pessoa com maior capacidade de aprendizado. ${ }^{3}$

A terceira função do casamento é o compartilhamento de bens públicos (coletivos). Os bens de consumo de uma família, em geral, são não rivais, e ambos os participantes da família podem compartilhá-los. São exemplos o desfrute dos filhos, compartilhar a mesma casa, compartilhar o mesmo automóvel e a troca de conhecimento e/ou informação. Segundo Lazear e Robert (1980), dois indivíduos podem quase que dobrar seu poder de compra ao se unirem. Adicionalmente, existem evidências de que as mães se preocupam mais com o bem-estar dos filhos do que os pais e, por isso, elas contribuem mais com bens públicos familiares, mesmo em um cenário de cooperação ou de problema dos caronas (Friedberg; Stern, 2004).

A quarta função do núcleo familiar é a partilha dos riscos. Por exemplo, suponha que um dos cônjuges se afasta do mercado de trabalho por motivo de doença ou demissão. Então, o outro indivíduo pode manter um nível de consumo satisfatório para ambos (ou pode minimizar as perdas no consumo) até que o cônjuge esteja recuperado da doença ou encontre outro emprego (Weiss, 1997; Browning et al., 2014).

3 As evidências desses arranjos de crédito no casamento são reveladas no momento do divórcio, quando o cônjuge que se dedicava a trabalhos domésticos deseja parte dos ganhos do cônjuge que foi apoiado durante o período de estudo e trabalho no mercado (Borenstein; Courant, 1989). 
Browning et al. (2014) mostram que o estado civil está fortemente correlacionado com a repartição do tempo de trabalho e os salários que os indivíduos recebem. Assim, em comparação com os solteiros, homens casados trabalham mais no mercado e têm salários maiores, enquanto as mulheres casadas trabalham menos no mercado, recebendo salários menores. Para Browning et al. (2014), esse padrão pode ser o resultado de dois efeitos diferentes. Primeiro, a divisão de trabalho entre os parceiros casados. Em segundo lugar, a seleção para o casamento, segundo a qual os homens dispostos (ou com possibilidade) a se casar possuem salários mais elevados e detêm alto potencial no mercado de trabalho, já as mulheres mais dispostas ao casamento possuem baixos salários e baixo potencial para o mercado de trabalho. Os autores encontraram que para todos os estados conjugais os homens trabalham mais do que as mulheres no mercado e as mulheres fazem mais tarefas domésticas do que os homens. Del Boca e Flinn (2013), por exemplo, concluem que, em geral, os cônjuges tomam suas decisões de alocação de tempo, em relação ao mercado de trabalho e tarefas domésticas, de maneira eficiente.

Com relação aos salários, Browning et al. (2014) apontam que as diferenças da participação de homens e mulheres casados no mercado de trabalho está relacionada às vantagens comparativas do casal. Os resultados mostram que os homens casados têm consistentemente maiores salários que seus pares solteiros, ao passo que o mesmo não é observado para mulheres. Sendo assim, seria mais vantajoso para o casal a mulher realizar as tarefas domésticas e os homens obterem remuneração no mercado de trabalho.

Quanto aos atributos individuais, Browning et al. (2014) concluem que os indivíduos escolhem seu cônjuge com base em seus próprios atributos e nos de seu parceiro. As interações dos atributos individuais geram ganhos mútuos no casamento. Por exemplo, um homem educado pode beneficiar-se mais de casar com uma mulher educada do que um homem menos educado, que pode até se ressentir em ter uma esposa mais educada do que ele. Da mesma forma, um casamento em que ambos os parceiros são semelhantes na idade pode criar ganhos mais elevados do que um casamento com uma grande discrepância em idades. Consequentemente, "os casamentos adequados" são mais propensos a se formar e menos prováveis de se dissolverem. Isso significa que os atributos observados de indivíduos casados podem ser muito diferentes dos atributos de homens e mulheres em geral. 
A discussão na literatura de alocação do tempo entre homens e mulheres dentro do núcleo familiar se estende sobre a questão da alocação desigual nas tarefas domésticas. E os canais geradores dessa desigualdade não estão plenamente identificados pela literatura empírica. Desse modo, há margem para discussão sobre a chamada dupla jornada de trabalho feminina, ${ }^{4}$ e diversas hipóteses são lançadas para explicar essa diferença, como, por exemplo, a hipótese cultural, a hipótese de que os homens trabalham mais duro, ou até mesmo a discriminação baseada em status e desvantagem associada a maternidade (Williams et al., 2013; Bielby; Bielby, 1988; Correl et al., 2007; Kleven et al., 2018). Há também a literatura que discute participação das mulheres entre mercado de trabalho e mercado doméstico sob a ótica dos estudos de gênero e feministas. Uma hipótese importante desse ramo da literatura recai sobre a possibilidade de a mulher assalariada utilizar parte de sua renda para contratação de um funcionário para exercer as tarefas domésticas, e dessa forma não geraria resultado igualitário entre os gêneros com relação às horas trabalhadas no domicílio (Kon, 2012; Madalozzo et al. 2010; Rangel, 2006; Dedecca, 2004; Wagner et al., 2005). Outros ramos da literatura são constituídos tanto pela análise de políticas públicas de cash bonus ${ }^{5}$ que buscam impactar tanto a alocação do tempo entre os cônjuges quanto a própria fertilidade do núcleo familiar (Bjorklund, 2006; Gauthier, 2007) quanto pela análise da relação com o gênero, o desemprego e a formalização do trabalho (Haussmann; Braz Golguer, 2016; Machado; Silva, 2014; Vieira Silva; Kassouf, 2013; Oliveira et al. 2011).

Cabe destacar o trabalho de Correl et al. (2007), onde os autores realizaram um experimento em laboratório e um estudo auditado para empregadores com objetivo de testar a hipótese penalidade sobre os salários e avaliações de performance sobre as mulheres que têm filhos. Os resultados mostram que as mães foram penalizadas em diversas medidas, incluindo na competência percebida e no salário inicial recomendado. Os homens não foram penalizados, e às vezes se beneficiaram por serem pais.

Kleven et al. (2018) argumentam que mesmo com a convergência temporal entre os gêneros, a maior parte da desigualdade salarial entre os gê-

4 A dupla jornada ocorre quando a mulher possui trabalho remunerado no mercado, e, além disso, trabalha dentro do domicílio em serviços domésticos.

5 Essas políticas são conhecidas por diversas nomenclaturas, como, por exemplo, Paternity Bonus, Baby Bonus, Cash Bonus etc. A ideia central de tais políticas é influenciar, por exemplo, a alocação, ou a fertilidade do núcleo familiar através da transferência de renda. 
neros deve-se aos filhos. Os autores utilizaram dados para a Dinamarca, de 1980 a 2013 e a metodologia de Event Study. Então, a chegada dos filhos cria um diferencial de rendimentos de aproximadamente $20 \%$ no longo prazo. Então, os child penalties afetam a dinâmica sobre ocupação, a promoção, e a simpatia da firma sobre a mulher, em relação ao homem. E uma possível explicação para a persistência da penalidade por ser mãe é a transmissão intergeracional dos pais para as filhas (mas não para os filhos), de modo a influenciar as preferências das meninas sobre questões familiares e de suas carreiras profissionais.

Queiroz e Aragon (2015), através do método de heckit e double hurdle, para dados da PNAD 2011, encontraram os determinantes da alocação do tempo para as mulheres brasileiras. Um dos resultados destacados pelos autores foi que as mulheres chefes de família têm mais chance de trabalhar (devido ao papel de provedora do lar), enquanto as mulheres casadas registram menor propensão ao trabalho. Logo, existe influência do matrimônio sobre a alocação do tempo, de modo que este reforça o papel da mulher como dona de casa. Além disso, as horas dedicadas ao trabalho doméstico pelas mulheres com filhos pequenos reduzem tanto a chance de trabalho quanto o número de horas trabalhadas.

Em termos da dinâmica da estrutura domiciliar, entre aos anos de 1970 a 2014, os dados mostram um declínio no número de famílias formadas por cônjuges nos Estados Unidos da América (Lundberg et al., 2016; Blau; Winkler, 2018). Segundo Blau e Winkler (2018), esse declínio do casamento formal está relacionado a diversas forças, tais como: a) econômicas (aumento da participação das mulheres na força de trabalho, seu nível de escolaridade, o compromisso profissional e salários combinados com a diminuição dos salários reais para homens menos qualificados); b) tecnológica (tecnologia reprodutiva, e a tecnologia doméstica); c) sociais (atitudes sobre sexo pré-marital e fecundidade não casada) e d) legais (mudanças nas leis de divórcio). E, além disso, o declínio nas famílias formadas por cônjuges foi acompanhado por um aumento considerável nas famílias monoparentais ${ }^{6}$ e de famílias plurais ${ }^{7}$. Segundo Winkler et al. (2005), outra

6 A família monoparental é formada por apenas um pai ou uma mãe. Ou seja, é aquela família em que apenas um dos pais de uma criança arca com todas as responsabilidades da criação do filho.

7 Famílias plurais, também chamadas de famílias abrangentes ou coabitadas, em que coabitam com a família nuclear avós, tios, netos de gravidez precoce etc. 
fonte de mudança significativa na estrutura domiciliar deve-se ao aumento da contribuição financeira das esposas nas famílias em que ambos os cônjuges trabalham. Nesse sentido, um dos fatores que contribuem para a diminuição do nível de renda entre os cônjuges é que os casais são cada vez mais propensos a se casar com aqueles que são semelhantes a si mesmos em termos de escolaridade. E esse padrão superior de remuneração das esposas afeta a relação de força de barganha dentro do domicílio.

À medida que a mulher obtém maiores salários no mercado de trabalho, esse maior nível de rendimento pode gerar instabilidade na união conjugal. Silva e Lazo (2010) estudaram as possíveis relações entre o aumento da inserção das mulheres no mercado de trabalho e as tendências do divórcio no Brasil no período de 1992 a 2007. Os autores utilizaram o conceito de que o risco da dissolução do matrimônio, dada a inserção da mulher no mercado de trabalho, se dá pelo desire effect (efeito do desejo), que se refere ao efeito do trabalho feminino na qualidade da união e na satisfação da mulher em permanecer unida ao cônjuge, e pelo opportunity effect (efeito da oportunidade), que investiga em que sentido a independência financeira feminina, conquistada pelo trabalho, poderia influenciar na decisão da mulher em deixar uma relação insatisfatória. Eles concluem que a maior parte dos divórcios ocorreu pelo efeito da oportunidade, adquirida pela independência financeira. Nessa mesma linha, Canêdo-Pinheiro et al. (2008) apontam que o aumento na taxa de divórcios no Brasil é resultado da diminuição da diferença de renda entre homens e mulheres. Adicionalmente, ambientes urbanos ou metropolitanos favorecem a dissolução dos matrimônios, bem como a maior educação da mulher e o desemprego masculino. Em contrapartida, filhos pequenos e a divisão das tarefas domésticas entre os cônjuges tendem a estabilizar a relação conjugal.

A literatura apresenta uma lacuna no que se refere ao papel do casamento sobre as variáveis de alocação de tempo dos cônjuges e mercado de trabalho para o Brasil. Sendo assim, este artigo procura contribuir para essa literatura ao estimar as diferenças entre casados e solteiros na divisão das tarefas produtivas e domésticas para cada gênero no Brasil através de métodos econométricos recentemente sugeridos pela literatura especializada, de modo a minimizar o viés das estimativas para amostras complexas através do escore de propensão. 


\section{Dados e metodologia}

Utilizamos dados da Pesquisa Nacional por Amostra de Domicílios (PNAD) de 2015. ${ }^{\circ}$ O questionário descreve a condição de cônjuge como sendo a pessoa que vivia conjugalmente com o chefe de família (referência da unidade familiar), existindo ou não o vínculo matrimonial. Logo, o cônjuge é definido como o companheiro vivendo no mesmo domicílio, independentemente de contrato de união. Sob a ótica da teoria econômica do casamento, consideramos tais indivíduos como "casados" ainda que não exista vínculo matrimonial formal. A utilização da PNAD de 2015 deve-se ao fato de esta ser a base mais recente disponível.

O questionário da PNAD foi aplicado a 356.904 pessoas em 117.939 unidades domiciliares, distribuídas pelas Unidades da Federação. Restringimos a nossa amostra de modo a trabalhar apenas com pessoas identificadas como chefe de família ou cônjuge, que são necessariamente maiores de idade e que possuíam emprego na semana de referência. Eliminamos da amostra funcionários públicos e militares ${ }^{9}$, bem como pessoas que não tinham a escolaridade informada. A primeira amostra (Amostra 1) é composta por 104.542 observações, entre as quais 61.688 correspondem a homens e 42.854 correspondem a mulheres. Posteriormente restringimos essa amostra de modo a incluir apenas os homens com esposas que exercem atividade no mercado de trabalho. A segunda amostra (Amostra 2) totaliza 23.597 observações.

Os modelos econométricos são estimados para as duas amostras distintas. Na Amostra 1 a variável de tratamento é uma dummy que identifica se o indivíduo possui cônjuge ou não. $\mathrm{Na}$ Amostra 2 a variável de tratamento é uma dummy que identifica se a esposa possui remuneração (de todos os trabalhos) maior do que o marido ou não.

As variáveis dependentes consideradas são o salário principal, a soma de todos os salários, a jornada semanal do trabalho principal, a jornada semanal de todos os trabalhos, a probabilidade de ter mais de um emprego, a probabilidade de exercer atividades domésticas e a jornada semanal de

8 A PNAD é uma pesquisa anual de corte transversal que adota um plano amostral complexo, com estratificação e conglomerados, representativa da população brasileira. A PNAD é realizada pelo Instituto Brasileiro de Geografia e Estatística (IBGE), que coleta informações sobre características socioeconômicas e demográficas de indivíduos e domicílios.

9 Também foram estimados os modelos incluindo os funcionários públicos e militares. As diferenças nos resultados são reportadas na seção 5 . 
trabalho doméstico. As informações relativas à atividade dos indivíduos são obtidas através da resposta a perguntas sobre o seu trabalho na semana de referência, podendo incluir trabalho formal e informal. Entretanto, não é possível identificar qual o tipo de atividade doméstica foi realizada pelo entrevistado, podendo incluir cuidado com os filhos ou outras tarefas domésticas.

Tabela 1 Caracterização das variáveis empregadas

\begin{tabular}{|c|c|}
\hline Variáveis & Caracterização \\
\hline \multicolumn{2}{|l|}{ Variáveis de tratamento } \\
\hline Casado & 1 se possui cônjuge, 0 caso contrário. \\
\hline Mulheres com salário maior & $\begin{array}{l}1 \text { se mulher possui salário de todos os trabalhos } \\
\text { maior que o homem, } 0 \text { caso contrário. }\end{array}$ \\
\hline \multicolumn{2}{|l|}{ Variáveis dependentes } \\
\hline Salário principal & Rendimento mensal no trabalho principal. \\
\hline Salário de todos os trabalhos & Rendimento mensal em todos os trabalhos. \\
\hline Jornada principal & Horas trabalhadas por semana no trabalho principal. \\
\hline Jornada de todos os trabalhos & Horas trabalhadas por semana em todos os trabalhos. \\
\hline Mais de um emprego & 1 se possui mais de um emprego, 0 caso contrário. \\
\hline Tarefa doméstica & 1 se executa tarefas domésticas, 0 caso contrário. \\
\hline Jornada doméstica & Horas destinadas a tarefas domésticas por semana. \\
\hline \multicolumn{2}{|l|}{ Covariáveis } \\
\hline Cor & 1 se for branco ou amarelo, 0 caso contrário. \\
\hline Área urbana & 1 se morar em área urbana, 0 caso contrário. \\
\hline Idade & Idade em anos. \\
\hline Anos de estudo & Número de anos que estudou. \\
\hline Número de filhos & Número de filhos que possui. \\
\hline Carteira assinada & 1 se possui carteira assinada, 0 caso contrário. \\
\hline Trabalhador doméstico & 1 se é trabalhador doméstico, 0 caso contrário. \\
\hline Conta própria & 1 se trabalha por conta própria, 0 caso contrário. \\
\hline Empregador & 1 se é empregador, 0 caso contrário. \\
\hline Estados & Dummies para cada Unidade da Federação. \\
\hline
\end{tabular}

Em todas as regressões utilizamos o logaritmo das variáveis de salário e jornada de trabalho, incluímos variáveis de controle para características individuais e da família, tais como idade, cor, anos de estudo e número de filhos, além de características do trabalho, como dummies identificando se 
o indivíduo possui carteira assinada, se é trabalhador doméstico, se trabalha por conta própria ou se é empregador, e características demográficas, como dummies para área urbana ou rural e para as diferentes Unidades da Federação. A Tabela 1 descreve as variáveis empregadas.

A Tabela 2 contém as estatísticas descritivas, a média e o desvio padrão, separadas por amostra, para as variáveis utilizadas. Através da Tabela 2 podemos ver a forma como os homens e as mulheres alocam seu tempo entre o mercado de trabalho e as tarefas domésticas, bem como diferenças salariais e de características individuais e socioeconômicas.

Tabela 2 Estatísticas descritivas por gênero e amostra

\begin{tabular}{lrr|r|r|r|r}
\hline \multirow{2}{*}{ Variável } & \multicolumn{3}{r}{ Amostra 1 } & \multicolumn{2}{r}{ Amostra 2 } \\
\cline { 2 - 8 } & Média & $\begin{array}{r}\text { Homens } \\
\text { Desvio } \\
\text { padrão }\end{array}$ & Média & $\begin{array}{r}\text { Desvio } \\
\text { padrão }\end{array}$ & Média & $\begin{array}{r}\text { Desvio } \\
\text { padrão }\end{array}$ \\
\cline { 2 - 8 } Casado & 0,8640 & 0,3428 & 0,6966 & 0,4597 & - & - \\
\hline Mulheres com salário maior & - & - & - & - & 0,1693 & 0,3751 \\
\hline Salário principal & $2.035,63$ & $3.278,00$ & $1.384,81$ & $2.157,49$ & $2.207,06$ & $3.452,06$ \\
\hline Salário de todos 0s trabalhos & $2.086,17$ & $3.396,25$ & $1.418,19$ & $2.236,91$ & $2.266,17$ & $3.559,65$ \\
\hline Jornada principal & 42,13 & 11,42 & 36,41 & 13,46 & 42,84 & 11,03 \\
\hline Jornada de todos 0s trabalhos & 42,77 & 11,90 & 36,95 & 13,80 & 43,53 & 11,56 \\
\hline Mais de um emprego & 0,0303 & 0,1715 & 0,0288 & 0,1673 & 0,0322 & 0,1764 \\
\hline Tarefa doméstica & 0,5758 & 0,4942 & 0,9458 & 0,2265 & 0,6012 & 0,4897 \\
\hline Jornada doméstica & 5,94 & 7,73 & 20,10 & 13,10 & 5,98 & 7,50 \\
\hline Cor & 0,4141 & 0,4926 & 0,4465 & 0,4971 & 0,4387 & 0,4962 \\
\hline Área urbana & 0,8413 & 0,3654 & 0,9209 & 0,2699 & 0,9049 & 0,2933 \\
\hline Idade & 42,61 & 12,23 & 40,75 & 11,53 & 41,24 & 10,92 \\
\hline Anos de estudo & 9,03 & 4,34 & 10,32 & 4,05 & 9,78 & 4,07 \\
\hline Número de filhos & 1,1519 & 1,1423 & 1,1782 & 1,0730 & 1,2358 & 1,0698 \\
\hline Carteira assinada & 0,4442 & 0,4969 & 0,4600 & 0,4984 & 0,5054 & 0,5000 \\
\hline Trabalhador doméstico & 0,0120 & 0,1090 & 0,1971 & 0,3978 & 0,0106 & 0,1024 \\
\hline Conta própria & 0,3399 & 0,4737 & 0,2467 & 0,4311 & 0,2926 & 0,4550 \\
\hline Empregador & 0,0612 & 0,2397 & 0,0342 & 0,1817 & 0,0731 & 0,2604 \\
\hline Número de observações & & 61.688 & & 42.854 & & 23.597 \\
\hline & & & & & & \\
\hline
\end{tabular}

Notas: A Amostra 1 corresponde aos chefes de família e cônjuges, maiores de idade, estavam empregados na semana de referência e tinham escolaridade informada, com exceção dos funcionários públicos e militares. A Amostra 2 restringe a Amostra 1 de modo a incluir apenas os homens com esposas que 
exercem atividade profissional. Os valores do salário e jornadas de trabalho estão em termos absolutos, embora nas regressões sejam usados os logaritmos dessas variáveis.

Através das estatísticas para a Amostra 1, observamos que homens e mulheres alocam seu tempo de forma diferente entre tarefas domésticas e mercado de trabalho. Os homens trabalham em média 42 horas semanais no mercado de trabalho e 6 horas semanais nas tarefas domésticas. Já as mulheres alocam menos horas no mercado de trabalho, cerca de 37 horas por semana, mas gastam 20 horas semanais com tarefas domésticas. A mulheres alocam, portanto, três vezes mais tempo do que os homens para a realização dessas tarefas. Em relação ao total de horas trabalhadas (mercado de trabalho e trabalho doméstico), as mulheres gastam cerca de 57 horas semanais, e os homens, 48 horas por semana, corroborando com a percepção de uma dupla jornada feminina. Em torno de 57,6\% dos homens declararam ter exercido alguma tarefa doméstica na semana de referência enquanto para as mulheres esse percentual foi de $94,6 \%$.

O salário médio dos homens na atividade principal é de $\mathrm{R} \$ 2.035,63$, em contraste aos $\mathrm{R} \$ 1.384,81$ auferidos pelas mulheres. Embora estas tenham, em média, mais anos de estudo: cerca de 10,3 anos para as mulheres e 9 anos para os homens.

Em torno de $40 \%$ dos homens trabalham por conta própria ou são empregadores, enquanto $44 \%$ possuem carteira assinada. Apenas $1 \%$ dos homens são trabalhadores domésticos. Para as mulheres, em torno de $28 \%$ trabalham por conta própria ou são empregadoras, enquanto $46 \%$ possuem carteira assinada. $O$ percentual de mulheres que são trabalhadoras domésticas é de quase $20 \%$. A Amostra 1 é composta majoritariamente por residentes em área urbana, com percentual superior a $80 \%$. A idade média dos indivíduos é de 42,6 anos para os homens e 40,7 para as mulheres, sendo que as famílias têm em média 1,15 filhos. Cerca de $86 \%$ dos homens e 70\% das mulheres são casadas nessa amostra.

A Amostra 2 é restrita apenas aos homens com esposas que exercem atividade profissional. As características dessa amostra são semelhantes às dos homens da Amostra 1. Destaca-se que 17\% dos homens possuem salários inferiores aos de suas esposas.

Como a PNAD é uma pesquisa com desenho amostral complexo, para identificar adequadamente os diferenciais na alocação de tempo entre casados e solteiros utilizamos métodos de escore de propensão aplicados a amostras complexas. $O$ escore de propensão é a probabilidade de trata- 
mento condicional às características observadas na amostra. Esse método permite replicar características particulares de estudos randomizados em estudos observacionais. A distribuição das covariáveis observadas, condicional ao escore de propensão, será similar entre os indivíduos que sofreram tratamento e os que não sofreram. Assim, o único fator observável que diferencia os indivíduos dos grupos tratado e de controle é o tratamento, sendo que as médias de todas as outras covariáveis são semelhantes.

De acordo com DuGoff et al. (2014), combinar os pesos do escore de propensão com os pesos da amostra complexa é necessário para alcançar o menor viés na estimação dos coeficientes. Eles realizaram simulações de Monte Carlo onde o mínimo viés absoluto foi obtido ao estimar o propensity score weighting (PSW) utilizando a multiplicação entre os pesos do escore de propensão e os pesos da amostra complexa. Austin et al. (2016) criticaram a limitação da simulação de Monte Carlo de DuGoff et al. (2014) e criaram uma amostra complexa mais detalhada. Através das simulações, os autores recomendaram o uso dos pesos amostrais complexos nas regressões após o pareamento da amostra.

Neste artigo estimamos três modelos para cada variável dependente. $\bigcirc$ primeiro modelo é uma regressão linear ponderada pelos pesos amostrais complexos (OLS). O segundo modelo é o propensity score weighting (PSW), em que estimamos uma regressão linear ponderada utilizando como pesos a multiplicação entre os pesos da regressão logística do escore de propensão e os pesos da amostra complexa. Esse método se baseia nas recomendações de DuGoff et al. (2014). O terceiro modelo é o propensity score matching (PSM), em que primeiro pareamos as observações tratadas através do algoritmo de nearest neighbour e, então, estimamos uma regressão linear ponderada com os pesos da amostra complexa nas observações pareadas. Esse método é baseado nas recomendações de Austin et al. (2016).

Assim, os modelos econométricos estimados terão a seguinte especificação:

$$
Y_{i}=\alpha+\gamma T_{i}+\beta X_{i}+\varepsilon_{i}
$$

onde $Y_{i}$ corresponde às variáveis dependentes utilizadas neste estudo, que estão descritas no segundo painel da Tabela $1 ; T_{i}$ se refere às variáveis de tratamento, descritas no primeiro painel da Tabela 1 (se casado, para a Amostra 1, ou se a esposa recebe remuneração maior, para a Amostra 2); 
e $X_{i}$ são as covariáveis utilizadas nas regressões, expressas no terceiro painel da Tabela 1. Para cada uma das variáveis dependentes e das variáveis de tratamento estimamos os três modelos descritos no parágrafo anterior.

\section{Resultados}

Para avaliar as diferenças entre casados e solteiros na alocação de tempo dos indivíduos, estimamos os três modelos apresentados na seção anterior utilizando as variáveis descritas na Tabela 1 para as Amostras 1 e 2. A Tabela 3 apresenta o balanço das covariáveis após o pareamento pelo escore de propensão (modelo PSM) para a Amostra 1 para ambos os gêneros, enquanto a Tabela 4 apresenta as diferenças entre casados e solteiros nas variáveis dependentes de interesse utilizando a Amostra 1 e os três modelos estimados: OLS, PSW e PSM.

Após estudar as mudanças entre casados e solteiros na alocação de tempo dos homens e mulheres, verificamos se diferenças na produtividade dos indivíduos podem explicar parte desses resultados ou se eles refletem apenas uma divisão de trabalho por gênero. Para isso restringimos a amostra apenas para homens com esposas que possuem emprego (Amostra 2) e então avaliamos as mudanças na alocação de tempo para aqueles casais em que a mulher aufere maior salário. A Tabela 5 apresenta o balanço das covariáveis após o pareamento pelo escore de propensão nessa amostra, enquanto a Tabela 6 apresenta os resultados dos três modelos estimados. Todas as regressões reportadas excluem da amostra os funcionários públicos e militares, conforme mencionado na seção Dados. Os modelos também foram estimados incluindo esses indivíduos na amostra, sendo que os resultados, nesse caso, não mudaram em termos da inferência estatística, porém em geral os coeficientes são um pouco menores, possivelmente devido a maior rigidez salarial dessas profissões.

$\mathrm{Na}$ Tabela 3, através do p-valor para o teste de diferença de médias, é possível verificar que as médias dos grupos tratado e de controle após o pareamento não apresentam diferenças significativas entre as covariáveis, com exceção de algumas variáveis como idade e número de filhos para as mulheres. Essa diferença na idade e no número de filhos para mulheres casadas é usual na literatura especializada (Browning et al., 2014). Assim, em geral os indivíduos apresentam bom balanceamento em termos de va- 
riáveis observadas. As dummies de Unidade de Federação foram omitidas nessa tabela por considerações de espaço. Note que esse balanço das covariáveis se refere às regressões do modelo PSM expressas nas colunas 3 e 6 da Tabela 4.

Tabela 3 Balanço das covariáveis para a Amostra 1

\begin{tabular}{lrr|r|r|r|r|r}
\hline & $\begin{array}{r}\text { Média } \\
\text { tratado }\end{array}$ & $\begin{array}{r}\text { Desvio } \\
\text { padrão } \\
\text { tratado }\end{array}$ & $\begin{array}{r}\text { Média } \\
\text { controle }\end{array}$ & $\begin{array}{r}\text { Desvio } \\
\text { padrão } \\
\text { controle }\end{array}$ & $\begin{array}{r}\text { Diferen- } \\
\text { ça de } \\
\text { médias }\end{array}$ & Teste-T & P-valor \\
\hline Homens & & & & & & & \\
\hline Cor & 0,378 & 0,484 & 0,376 & 0,484 & 0,002 & 0,368 & 0,713 \\
\hline Área urbana & 0,847 & 0,353 & 0,854 & 0,353 & $-0,007$ & $-1,566$ & 0,117 \\
\hline Idade & 43,484 & 13,938 & 44,124 & 13,938 & $-0,641$ & $-3,438$ & 0,001 \\
\hline Anos de estudo & 8,922 & 4,501 & 8,904 & 4,501 & 0,019 & 0,311 & 0,756 \\
\hline Número de filhos & 0,270 & 0,656 & 0,270 & 0,656 & $-0,0001$ & $-0,014$ & 0,989 \\
\hline Carteira assinada & 0,395 & 0,490 & 0,398 & 0,490 & $-0,004$ & $-0,546$ & 0,585 \\
\hline Trabalhador doméstico & 0,022 & 0,140 & 0,020 & 0,140 & 0,002 & 1,212 & 0,225 \\
\hline Conta própria & 0,377 & 0,486 & 0,380 & 0,486 & $-0,003$ & $-0,459$ & 0,646 \\
\hline Empregador & 0,045 & 0,210 & 0,046 & 0,210 & $-0,001$ & $-0,466$ & 0,641 \\
\hline Mulheres & & & & & & & \\
\hline Cor & 0,409 & 0,492 & 0,414 & 0,492 & $-0,005$ & $-0,945$ & 0,345 \\
\hline Área urbana & 0,950 & 0,211 & 0,953 & 0,211 & $-0,003$ & $-1,460$ & 0,144 \\
\hline Idade & 43,539 & 11,491 & 43,257 & 11,491 & 0,282 & 2,283 & 0,022 \\
\hline Anos de estudo & 9,884 & 4,183 & 9,966 & 4,183 & $-0,081$ & $-1,806$ & 0,071 \\
\hline Número de filhos & 1,103 & 1,023 & 1,067 & 1,023 & 0,036 & 3,270 & 0,001 \\
\hline Carteira assinada & 0,444 & 0,498 & 0,452 & 0,498 & $-0,008$ & $-1,496$ & 0,135 \\
\hline Trabalhador doméstico & 0,240 & 0,423 & 0,233 & 0,423 & 0,007 & 1,575 & 0,115 \\
\hline Conta própria & 0,235 & 0,424 & 0,235 & 0,424 & 0,0005 & 0,101 & 0,919 \\
\hline Empregador & 0,024 & 0,151 & 0,023 & 0,151 & 0,0005 & 0,284 & 0,776 \\
\hline & & & & & & & \\
\hline
\end{tabular}

Notas: Os valores correspondem a amostra após o pareamento realizado pelo modelo PSM. As dummies de estado foram omitidas por considerações de espaço. A razão-T e o p-valor são relativos ao teste de diferença de médias. O grupo tratado refere-se a indivíduos casados. O grupo de controle é composto por não casados.

$\mathrm{Na}$ Tabela 4 observamos as diferenças entre casados e solteiros em todas as variáveis dependentes de interesse. Por economia de espaço, reportamos apenas os coeficientes da dummy que identifica se a pessoa possui cônjuge ou não. Cada coluna representa um dos três modelos estimados para os 
homens e mulheres. Podemos ver que os homens casados têm, em média, salários $7 \%$ maiores e trabalham, em média, 3\% a mais que seus pares solteiros, entretanto, não há nenhum efeito significativo do casamento no salário das mulheres, sendo que a jornada de trabalho das mulheres casadas é, em média, cerca de $5 \%$ menor. Também é menor a probabilidade de as mulheres terem mais de um emprego quando estão casadas.

Tabela 4 Diferenças de salário e alocação de tempo entre casados e solteiros

\begin{tabular}{|c|c|c|c|c|c|c|}
\hline \multirow[b]{2}{*}{ Variável dependente } & \multicolumn{3}{|r|}{ Homens } & \multicolumn{3}{|r|}{ Mulheres } \\
\hline & $\begin{array}{r}\text { OLS } \\
(1)\end{array}$ & $\begin{array}{r}\text { PSW } \\
(2)\end{array}$ & $\begin{array}{r}\text { PSM } \\
\text { (3) }\end{array}$ & $\begin{array}{r}\text { OLS } \\
(4)\end{array}$ & $\begin{array}{r}\text { PSW } \\
\text { (5) }\end{array}$ & $\begin{array}{r}\text { PSM } \\
(6)\end{array}$ \\
\hline Salário principal & $\begin{array}{r}0,073^{* * *} \\
(0,009)\end{array}$ & $\begin{array}{r}0,076^{* * *} \\
(0,009)\end{array}$ & $\begin{array}{r}0,071^{* * *} \\
(0,011)\end{array}$ & $\begin{array}{r}0,005 \\
(0,008)\end{array}$ & $\begin{array}{r}0,005 \\
(0,008)\end{array}$ & $\begin{array}{l}-0,001 \\
(0,009)\end{array}$ \\
\hline$N$ & 61.688 & 61.688 & 16.784 & 42.854 & 42.854 & 26.004 \\
\hline Salário de todos os trabalhos & $\begin{array}{r}0,070^{* * *} \\
(0,009)\end{array}$ & $\begin{array}{r}0,073^{* * *} \\
(0,009)\end{array}$ & $\begin{array}{r}0,069^{* * *} \\
(0,011)\end{array}$ & $\begin{array}{l}-0,001 \\
(0,008)\end{array}$ & $\begin{array}{l}-0,002 \\
(0,008)\end{array}$ & $\begin{array}{l}-0,007 \\
(0,009)\end{array}$ \\
\hline$N$ & 61.688 & 61.688 & 16.784 & 42.854 & 42.854 & 26.004 \\
\hline Jornada do trabalho principal & $\begin{array}{r}0,031^{* * *} \\
(0,006)\end{array}$ & $\begin{array}{r}0,032^{* * *} \\
(0,006)\end{array}$ & $\begin{array}{r}0,020^{* * *} \\
(0,008)\end{array}$ & $\begin{array}{r}-0,042^{* * *} \\
(0,006)\end{array}$ & $\begin{array}{r}-0,041^{* * *} \\
(0,006)\end{array}$ & $\begin{array}{r}-0,046^{* * *} \\
(0,007)\end{array}$ \\
\hline$N$ & 61.688 & 61.688 & 16.784 & 42.854 & 42.854 & 26.004 \\
\hline Jornada de todos os trabalhos & $\begin{array}{r}0,028^{* * *} \\
(0,006)\end{array}$ & $\begin{array}{r}0,029 * * * \\
(0,006)\end{array}$ & $\begin{array}{l}0,018^{* *} \\
(0,007)\end{array}$ & $\begin{array}{r}-0,047^{* * *} \\
(0,006)\end{array}$ & $\begin{array}{r}-0,047^{* * *} \\
(0,006)\end{array}$ & $\begin{array}{r}-0,051^{* * *} \\
(0,007)\end{array}$ \\
\hline$N$ & 61.688 & 61.688 & 16.784 & 42.854 & 42.854 & 26.004 \\
\hline Prob. de mais de um emprego & $\begin{array}{c}-0,004^{*} \\
(0,002)\end{array}$ & $\begin{array}{l}-0,004 \\
(0,002)\end{array}$ & $\begin{array}{l}-0,001 \\
(0,003)\end{array}$ & $\begin{array}{r}-0,007^{* * *} \\
(0,002)\end{array}$ & $\begin{array}{r}-0,007^{* * *} \\
(0,002)\end{array}$ & $\begin{array}{r}-0,006^{* * *} \\
(0,002)\end{array}$ \\
\hline$N$ & 61.688 & 61.688 & 16.784 & 42.854 & 42.854 & 26.004 \\
\hline Prob. de tarefa doméstica & $\begin{array}{r}-0,277^{* * *} \\
(0,006)\end{array}$ & $\begin{array}{r}-0,273^{* * *} \\
(0,006)\end{array}$ & $\begin{array}{r}-0,274^{* * *} \\
(0,008)\end{array}$ & $\begin{array}{r}0,014^{* * *} \\
(0,003)\end{array}$ & $\begin{array}{r}0,015^{* * *} \\
(0,003)\end{array}$ & $\begin{array}{r}0,013^{* * * *} \\
(0,003)\end{array}$ \\
\hline$N$ & 61.688 & 61.688 & 16.784 & 42.854 & 42.854 & 26.004 \\
\hline Jornada de tarefa doméstica & $\begin{array}{r}-0,834^{* * *} \\
(0,017)\end{array}$ & $\begin{array}{r}-0,825^{* * *} \\
(0,018)\end{array}$ & $\begin{array}{r}-0,834^{* * *} \\
(0,020)\end{array}$ & $\begin{array}{r}0,190^{* * *} \\
(0,011)\end{array}$ & $\begin{array}{r}0,196 * * * \\
(0,011)\end{array}$ & $\begin{array}{r}0,191^{* * *} \\
(0,012)\end{array}$ \\
\hline$N$ & 61.688 & 61.688 & 16.784 & 42.854 & 42.854 & 26.004 \\
\hline
\end{tabular}

Notas: As variáveis de controle foram omitidas por considerações de espaço. Os valores em parênteses são os desvios padrão dos coeficientes. Os símbolos ${ }^{*},{ }^{* *}{ }^{* * *}$ representam significância estatística de $10 \%, 5 \%$ e $1 \%$, respectivamente.

No que se refere a alocação de tempo dos casais dentro do domicílio, podemos ver que o casamento diminui a probabilidade de o homem exercer tarefas domésticas em cerca de 27 pontos percentuais, enquanto aumenta a 
probabilidade de a mulher exercer essas tarefas em cerca de 1,3 pontos percentuais. Já as horas despendidas em tarefas domésticas diminuem drasticamente para homens casados em cerca de $83 \%$, enquanto aumentam para as mulheres casadas em cerca de $19 \%$. Esses resultados podem ser reflexo de uma divisão de trabalho baseada no gênero ou então numa possível vantagem comparativa obtida pelos homens no mercado de trabalho, seja por fatores relativos a produtividade ou por discriminações salariais.

Os resultados encontrados na Tabela 4 mostram que os homens casados têm consistentemente maiores salários que seus pares solteiros, enquanto as mulheres casadas não têm salários maiores em relação às solteiras. Desse modo, corroboramos os resultados encontrados por Browning et al. (2014) com dados para o Brasil. Para avaliarmos se esses resultados refletem puramente uma divisão de trabalho de gênero, construímos uma amostra composta apenas por homens com esposas que trabalham (Amostra 2), e verificamos o que ocorre na alocação de tempo quando a mulher aufere um salário maior do que seu parceiro. A Tabela 5 apresenta o balanço das covariáveis no modelo PSM, cujas estimações correspondem à coluna 3 da Tabela 6.

Tabela 5 Balanço das covariáveis para a Amostra 2

\begin{tabular}{lrrrrrr|r|r|r}
\hline & $\begin{array}{r}\text { Média } \\
\text { tratado }\end{array}$ & $\begin{array}{r}\text { Desvio } \\
\text { padrão } \\
\text { tratado }\end{array}$ & $\begin{array}{r}\text { Média } \\
\text { controle }\end{array}$ & $\begin{array}{r}\text { Desvio } \\
\text { padrão } \\
\text { controle }\end{array}$ & $\begin{array}{r}\text { Diferen- } \\
\text { ça de } \\
\text { médias }\end{array}$ & Teste-T & P-valor \\
\hline Homens & & & & & & & \\
\hline Cor & 0,415 & 0,493 & 0,413 & 0,492 & 0,002 & 0,262 & 0,793 \\
\hline Área urbana & 0,908 & 0,289 & 0,914 & 0,280 & $-0,006$ & $-1,130$ & 0,259 \\
\hline Idade & 41,114 & 11,358 & 40,817 & 10,899 & 0,297 & 1,367 & 0,172 \\
\hline Anos de estudo & 9,748 & 4,114 & 9,907 & 3,983 & $-0,159$ & $-2,015$ & 0,044 \\
\hline Número de filhos & 1,189 & 1,098 & 1,157 & 1,026 & 0,031 & 1,503 & 0,133 \\
\hline Carteira assinada & 0,453 & 0,498 & 0,460 & 0,498 & $-0,007$ & $-0,700$ & 0,484 \\
\hline Trabalhador doméstico & 0,011 & 0,103 & 0,006 & 0,079 & 0,004 & 2,428 & 0,015 \\
\hline Conta própria & 0,345 & 0,476 & 0,343 & 0,475 & 0,002 & 0,190 & 0,849 \\
\hline Empregador & 0,038 & 0,191 & 0,039 & 0,194 & $-0,001$ & $-0,336$ & 0,737 \\
\hline
\end{tabular}

Notas: Os valores correspondem a amostra após o pareamento realizado pelo modelo PSM. As dummies de estado foram omitidas por considerações de espaço. A razão-Te o p-valor são relativos ao teste de diferença de médias. O grupo tratado refere-se a homens casados com esposas auferindo remuneração superior à do marido. O grupo de controle é composto por homens casados com mulheres atuantes no mercado recebendo remuneração inferior ou igual à de seus maridos. 
A última coluna da Tabela 5 apresenta o p-valor do teste de diferença de médias para as covariáveis entre o grupo controle e o grupo tratado, pareado através do PSM. Podemos ver que todas as covariáveis possuem médias semelhantes, de modo que os indivíduos foram adequadamente balanceados. As dummies das Unidades de Federação foram omitidas para economia de espaço.

Tabela 6 Diferenças de salário e alocação de tempo dos homens quando a esposa aufere salário maior

\begin{tabular}{lrrr}
\hline \multirow{2}{*}{ Variável dependente } & & & Homens \\
\cline { 2 - 4 } Salário principal & $\begin{array}{r}\text { OLS } \\
\mathbf{( 1 )}\end{array}$ & $\begin{array}{r}\text { PSW } \\
\mathbf{( 2 )}\end{array}$ & $\begin{array}{r}\text { PSM } \\
\mathbf{( 3 )}\end{array}$ \\
\hline $\boldsymbol{N}$ & $-0,415^{* * *}$ & $-0,429^{* * *}$ & $-0,410^{* * *}$ \\
\hline Salário de todos os trabalhos & $(0,012)$ & $(0,012)$ & $(0,015)$ \\
\hline $\boldsymbol{N}$ & 23.597 & 23.597 & 7.992 \\
\hline \multirow{2}{*}{ Jornada do trabalho principal } & $-0,428^{* * *}$ & $-0,442^{* * *}$ & $-0,421^{* * *}$ \\
& $(0,012)$ & $(0,012)$ & $(0,015)$ \\
\hline $\boldsymbol{N}$ & 23.597 & 23.597 & 7.992 \\
\hline \multirow{2}{*}{ Jornada de todos os trabalhos } & $-0,066^{* * *}$ & $-0,074^{* * *}$ & $-0,067^{* * *}$ \\
& $(0,008)$ & $(0,009)$ & $(0,010)$ \\
\hline $\boldsymbol{N}$ & 23.597 & 23.597 & 7.992 \\
\hline \multirow{2}{*}{ Prob. de mais de um emprego } & $-0,056^{* * *}$ & $-0,064^{* * *}$ & $-0,059^{* * *}$ \\
\hline $\boldsymbol{N}$ & $(0,008)$ & $(0,009)$ & $(0,010)$ \\
\hline \multirow{2}{*}{ Prob. de tarefa doméstica } & 23.597 & 23.597 & 7.992 \\
\hline $\boldsymbol{N}$ & $-0,023^{* * *}$ & $-0,023^{* * *}$ & $-0,019^{* * *}$ \\
\hline \multirow{2}{*}{ Jornada de tarefa doméstica } & $(0,002)$ & $(0,003)$ & $(0,004)$ \\
\hline $\boldsymbol{N}$ & 23.597 & 23.597 & 7.992 \\
\hline & $0,064^{* * *}$ & $0,066^{* * *}$ & $0,062^{* * *}$ \\
\hline
\end{tabular}

Nota: As variáveis de controle foram omitidas por considerações de espaço. Os valores em parênteses são os desvios padrão dos coeficientes. Os símbolos ${ }^{*},{ }^{* *} e^{* * *}$ representam significância estatística de $10 \%, 5 \%$ e $1 \%$, respectivamente. 
A Tabela 6 apresenta as diferenças na alocação de tempo do homem quando a mulher aufere um salário maior. Esses homens ganham, em média, $41 \%$ a menos, e sua jornada de trabalho é reduzida em cerca de $6 \%$, número semelhante à redução da jornada de trabalho das mulheres na Tabela 4. Também, os homens cujas esposas recebem maiores salários têm probabilidade mais baixa de possuir mais de um emprego, cerca de 2 pontos percentuais menor.

As diferenças na alocação de tempo dos homens dentro do domicílio quando as mulheres auferem maiores salários se alteram em relação aos resultados anteriores expressos na Tabela 4. Nesse caso, os homens aumentam sua probabilidade de exercer tarefa doméstica em cerca de 6 pontos percentuais, enquanto a jornada de trabalho doméstico aumenta em cerca de $20 \%$ em relação aos demais homens casados, número também semelhante ao aumento das mulheres na Tabela 4. Nesse sentido, os efeitos na alocação de tempo encontrados para as mulheres na Tabela 4 são sentidos para os homens no caso em que a mulher aufere maior salário, o que reflete o fato de que parte da alocação de tempo dos casais é baseada na vantagem comparativa. Entretanto, apesar de ocorrer um aumento na participação do homem nas tarefas domésticas de cerca de $20 \%$ quando a mulher aufere maior salário, isso representa um aumento da jornada doméstica média de 5,98 para 7,17 horas semanais, enquanto a jornada doméstica média das mulheres cai de 20,10 para 18,97 horas semanais, sendo ainda consideravelmente superior à dos homens. Assim, apesar de parte da alocação de tempo dos casais ser baseada na vantagem comparativa, a mulher continua sendo suscetível ao problema da dupla jornada.

Dessa forma, assim como Del Boca e Flinn (2013), podemos concluir que as decisões de alocação de tempo dos cônjuges em relação ao mercado de trabalho são afetadas pela vantagem comparativa do casal. Ou seja, o cônjuge que recebe o maior salário é aquele que dedica mais horas ao mercado de trabalho, ao passo que o cônjuge menos produtivo aumenta suas horas dedicadas às tarefas domésticas, embora isso não mitigue o problema da dupla jornada das mulheres. Conforme vimos, uma das funções do casamento é justamente dividir o trabalho a fim de tirar proveito das vantagens comparativas. A união proporciona aos seus membros a opção de utilizar melhor o capital humano. Assim, o casal avalia as vantagens comparativas e aloca de forma apropriada quem ofertará mais horas no mercado e quem dedicará mais horas para as tarefas domésticas. 


\section{Considerações finais}

O presente estudo analisou as diferenças entre casados e solteiros no salário e na alocação de tempo, procurando entender como o casamento pode alterar a distribuição de salários e a jornada de trabalho entre homens e mulheres, especialmente no caso em que as mulheres auferem salários superiores aos de seus respectivos cônjuges, o que pode contribuir para entender o papel da vantagem comparativa do casal na alocação de tempo e mercado de trabalho. Esta análise foi realizada através de métodos baseados nas recomendações recentes da literatura sobre escore de propensão aplicado a amostras complexas, de modo a garantir a robustez e solidez dos resultados, bem como a minimização do viés nas estimativas.

Os resultados demonstram que enquanto homens casados têm salários em média superiores aos dos solteiros, o mesmo não acontece para as mulheres, evidenciando que os resultados discutidos por Browning et al. (2014) também estão presentes no Brasil. Já as atividades domésticas são afetadas pela estrutura conjugal, uma vez que homens casados têm probabilidade menor de exercer tarefas domésticas, bem como diminuem consideravelmente a jornada desse tipo de trabalho, ao contrário do que acontece com as mulheres casadas, que tendem a aumentar tanto a probabilidade quanto a jornada de tarefa doméstica. Por fim, quando as mulheres recebem salários superiores aos seus respectivos cônjuges, a jornada das tarefas domésticas e a probabilidade de exercê-las aumenta para o homem, de modo que parte da alocação de tempo do casal é baseada na vantagem comparativa, embora essa jornada doméstica do homem não iguale a da mulher, ou seja, o problema da dupla jornada das mulheres não é eliminado.

Com isso este artigo contribui para a literatura de diferenciais salariais e de alocação de tempo entre os gêneros estimando empiricamente as diferenças entre casados e solteiros na divisão das tarefas produtivas e domésticas para cada gênero no Brasil. Cabe destacar que esta pesquisa também emprega métodos econométricos recentemente sugeridos pela literatura especializada, de modo a minimizar o viés das estimativas para amostras complexas através do escore de propensão. 


\section{Referências}

AUSTIN, P. C.; JEMBERE, N.; CHIU, M. (2016). Propensity Score Matching and Complex Surveys. Statistical Methods in Medical Research, v. 0, pp.1-18.

BALBINOTTO NETO, G. (1992). A teoria econômica do casamento e do divórcio. Análise Econômica (UFRGS), v. 10, n. 18, pp.125-141.

BECKER, G. S. (1973). Theory of Marriage: Part I. Journal of Political Economy, v. 81 (4), p. 300-310.

BIELBY, D. D.; BIELBY, W. T. (1988). She Works Hard for the Money: Household Responsibilities and the Allocation of WorkEffort. American Journal of Sociology, v. 93, n. 5, pp. 1031-1059, Mar. 1988.

BJORKLUND, A. (2006). Does Family Policy Affect Fertility? Lessons from Sweden. Journal of Population Economics, 19(1), 3-24.

BLAU, F. D.; WINKLER, A. E. (2018). Women, Work, and Family. In: AVERETT; S. L.; ARGYS, L. M.; HOFFMAN, S. D. (Ed.). Oxford Handbook of Women and the Economy. , Oxford University Press.

BORENSTEIN, S.; COURANT, P. N. (1989). How to Carve a Medical Degree: Human Capital Assets in Divorce Settlements. American Economic Review, v. 79, n. 5, 992-1009.

BROWNING, M.; CHIAPPORI, P. A.; WEISS, Y. (2014). Family Economics. Cambridge, UK: Cambridge University Press.

CANÊDO-PINHEIRO, M.; LIMA, L. R.; MOURA, R. L. (2008). Fatores econômicos e incidência de divórcios: evidências com dados agregados brasileiros. In: XXXVI ENCONTRO NACIONAL DE ECONOMIA.

CORREL, S. J.; BENARD, S.; PAIK, I. (2007). Getting a Job: Is There a Motherhood Penalty? 1. American Journal of Sociology, 112(5), 1297-1339.

DEDECCA, C. S. Tempo, trabalho e gênero. In: COSTA, A. A.; OLIVEIRA, E. M.; LIMA, M. E. B.; SOARES, V. (Org.) (2004). Reconfiguração das relações de gênero e trabalho. CUT Brasil (Ed.).

DEL BOCA, D.; FLINN, C. (2013). Household Behavior and the Marriage Market. Journal of Economic Theory, 150, 515-550.

DUGOFF, E. H.; SCHULER, M.; STUART, E. A. (2014). Generalizing Observational Study Results: Applying Propensity Score Methods to Complex Surveys. Health Services Research 49 (1), 284-303.

FRIEDBERG, L.; STERN, S. (2004). Marriage, Divorce, and Asymmetric Information. University of Virginia, Department of Economics, Charlottesville. Unpublished manuscript.

GAUTHIER, A. (2007). The Impact of Family Policies on Fertility in Industrialized Countries: A Review of the Literature. Popul Res Policy Rev. 26:323-346.

GRONAU, R. (1986). Home Production a Survey. In: ASHENFELTER, O.; LAYARD, R. (Ed.). Handbook of Labor Economics. v. 1, pp. 273-304. Amsterdam: Elsevier Science Publishers.

HAUSSMANN, S.; BRAZ GOLGUER, A. (2016). Shrinking gender Wage Gaps in the Brazilian Labor Market: An Application of the APC Approach. Nova Economia, v. 26, n. 2.

KLEVEN, H.; LANDAIS, C.; SOGAARD, J. E. (2018). Children and Gender Inequality: Evidence 
from Denmark. NBER Working Paper Series. Working Paper 24219.

KON, A. A economia política do gênero: determinantes da divisão do trabalho. Revista de Economia Política, v. 22, n. 3, p. 89-109, 2002.

KORENMAN, S.; NEUMARK, D. (1991). Does Marriage Really Make Men More Productive? Journal oh Human Resources, 26(2), 282-307.

LAZEAR, E. P.; ROBERT, T. M. (1980). Family Size and the Distribution of Real per Capita Income. The American Economic Review, v. 70, n. 1, pp. 91-107.

LUNDBERG, S.; POLLAK, R. A.; STEARNS, J. E. (2016). Family Inequality: Diverging Patterns in Marriage, Cohabitation, and Childbearing. Journal of Economic Perspectives 30, n. 2, Spring: 79-102.

MACHADO, D. C.; SILVA, A. F. da (2014). Um indicador de não satisfação no trabalho e a mobilidade do Mercado de trabalho: um estudo para homens e mulheres. Nova Economia, v. 24, n. 1.

MADALOZZO, R.; MARTINS, S. R.; SHIRATORI, L (2010). Participação no mercado de trabalho e no trabalho doméstico: homens e mulheres têm condições iguais? Rev. Estud. Fem. [on-line]. v. 18, n. 2, pp. 547-566.

MAN, Y. K.; SULLIVAN, O.; GERSHUNY, J. (2011). Gender Convergence in Domestic Work: Discerning the Effects of Interactional and Institutional Barriers from Large-scale Data. Sociology, 45, 234-251.

OLIVEIRA, P. R. de; SCORZAFAVE, L. G.; PAZELLO, E. T. Desemprego e inatividade nas metrópoles brasileiras: as diferenças entre homens e mulheres. Nova Economia, v. 19, n. 2, 2011.

QUEIROZ, V. dos S.; ARAGON, J. A. O. (2015). Alocação de tempo em trabalho pelas mulheres brasileiras. Estud. Econ., v. 45, n. 4, pp. 787-819.

RANGEL, Marcos A. (2006). Alimony Rights and Intrahousehold Allocation of Resources: Evidence from Brazil. The Economic Journal, v. 116, p. 627-658.

SAYER, L. C. (2010). Trends in Housework. In: TREAS J.; DROBNIC, S. (Ed.). Dividing the Domestic: Men, Women, and Household Work in Cross-National Perspective. Stanford: Stanford University Press. pp. 19-38.

SILVA, M. F. B da; LAZO, A. V. (2008). A participação dos cônjuges no mercado de trabalho e o divórcio no Brasil. Economia e Desenvolvimento, Recife, v. 7, p. 38-60.

VIEIRA SILVA, N. de D.; KASSOUF, A. L. (2013). Mercados de trabalho formal e informal: uma análise da discriminação e da segmentação. Nova Economia, v. 10, n. 1.

WAGNER, A.; PREDEBON, J.; MOSMANN, C.; VERZA, F. (2005). Compartilhar tarefas? Papéis e funções de pai e mãe na família contemporânea. Psicologia: Teoria e Pesquisa, v. 21, n. 2, p. 181-186.

WEISS, Y. (1997). The Formation and Dissolution of Families: Why Marry? Who Marries Whom? And What Happens Upon Divorce. Rosenzweig, M. R.; Stark, O. (Ed.). Handbook of Population and Family Economics, v. 1A, p. 81-123.

WILLIAMS, J. C.; BLAIR-LOY, M.; BERDAHL, J. L. (2013). Cultural Schemas, Social Class, and Flexibility Stigma. Journal of Social Issues, v. 69, n. 2, pp.209-234. 
WINKLER, A. E.; McBRIDE, T. D.; ANDREWS, C. (2005). Wives Who Outearn Their Husbands: A Transitory or Persistent Phenomenon for Couples? Demography 42, n. 3 (August): 523-535.

\section{Sobre os autores}

Daniel de Abreu Pereira Uhr - daniel.uhr@gmail.com Universidade Federal de Pelotas, Pelotas, Rio Grande do Sul, Brasil. ORCID: https://orcid.org/0000-0001-8370-0317.

Regis Augusto Ely - regisaely@gmail.com Universidade Federal de Pelotas, Pelotas, Rio Grande do Sul, Brasil. ORCID: https://orcid.org/0000-0002-2468-3761.

Renata Pereira Cardoso - renata.cardoso.rs@gmail.com Doutoranda no Programa de Pós-Graduação em Economia do Desenvolvimento da Pontifícia Universidade Católica do Rio Grande do Sul, Porto Alegre, Rio Grande do Sul, Brasil. ORCID: https://orcid.org/0000-0001-6775-6222.

Júlia Gallego ZieroUhr-zierouhr@gmail.com

Universidade Federal de Pelotas, Pelotas, Rio Grande do Sul, Brasil. ORCID: https://orcid.org/0000-0003-4981-848X.

\section{Sobre o artigo}

Recebido em 11 de agosto de 2017. Aprovado em 16 de julho de 2018. 